\title{
Machine Learning-enhanced Receive Processing for MU-MIMO OFDM Systems
}

\author{
Mathieu Goutay* ${ }^{*}$, Fayçal Ait Aoudia*, Jakob Hoydis ${ }^{\dagger}$, and Jean-Marie Gorce \\ *Nokia Bell Labs, Paris-Saclay, 91620 Nozay, France \\ ${ }^{\dagger}$ NVIDIA, 06906 Sophia Antipolis, France \\ $\ddagger$ Université de Lyon, INSA Lyon, Inria, CITI, 69100 Villeurbanne, France \\ \{mathieu.goutay, faycal.ait_aoudia\}@nokia.com,jhoydis@nvidia.com, jean-marie.gorce@insa-lyon.fr
}

\begin{abstract}
Machine learning (ML) can be used in various ways to improve multi-user multiple-input multiple-output (MUMIMO) receive processing. Typical approaches either augment a single processing step, such as symbol detection, or replace multiple steps jointly by a single neural network (NN). These techniques demonstrate promising results but often assume perfect channel state information (CSI) or fail to satisfy the interpretability and scalability constraints imposed by practical systems. In this paper, we propose a new strategy which preserves the benefits of a conventional receiver, but enhances specific parts with ML components. The key idea is to exploit the orthogonal frequency-division multiplexing (OFDM) signal structure to improve both the demapping and the computation of the channel estimation error statistics. Evaluation results show that the proposed ML-enhanced receiver beats practical baselines on all considered scenarios, with significant gains at high speeds.
\end{abstract}

\section{INTRODUCTION}

Future generations of wireless networks will need to handle the growing demand for connectivity. At the physical layer, multi-user multiple-input multiple-output (MU-MIMO) is a promising technique to increase the number of users that can be served simultaneously. Linear methods are often used to decrease the computational complexity of the receive processing, but they achieve poor performance on realistic channels. It is therefore crucial to find new solutions that both satisfy the constraints of practical deployments and the performance requirements of future wireless communication systems.

Motivated by the successes of machine learning (ML) when applied to the physical layer [2], two ML-based approaches have been proposed to improve MIMO reception. The first one consists in augmenting a single processing step of a conventional MIMO receiver using a neural network (NN). This strategy has been applied to separately improve channel estimation [3], [4], equalization [5-7], and demapping [8]. Although encouraging results have been shown, the proposed solutions usually require perfect channel state information (CSI), remain too complex for practical deployments, or are designed for single-input single-output (SISO) systems only.

The second approach is to replace multiple receive components by a single NN. For example, [9] demonstrates strong results by replacing the channel estimation, equalization, and demapping steps by a convolutional neural network (CNN)

Work carried out while J. Hoydis was with Nokia Bell Labs. coupled with a so-called transformation layer. The main advantage is that the NN is directly optimized to improve the accuracy of the estimated bits, but the counterpart is that the number of connected users is dictated by the NN architecture.

In this paper, we introduce a new strategy which aims to combine the advantages of both approaches while avoiding their shortcomings. The key idea is to use several ML components to enhance specific parts of a conventional MU-MIMO architecture. More precisely, the orthogonal frequency-division multiplexing (OFDM) signal structure is exploited by multiple CNNs to improve two receive processing steps. The first one is the computation of the channel estimation error second order statistics, that the CNNs are able to learn during training. The second one is the demapping, which is carried out by a CNN processing the entire OFDM time-frequency grid instead of individual resource elements. All CNNs are jointly optimized to maximize the information rate of the transmission [10].

The proposed ML-enhanced receiver is benchmarked against two conventional receivers, the second one having perfect CSI. A 3GPP-compliant channel model was considered, with two pilot configurations and users moving at speeds ranging from 0 to $130 \mathrm{~km} \mathrm{~h}^{-1}$. The results indicate that the ML-enhanced receiver beats the baseline on every considered scenario and enables gains that increase with the user speeds.

Notations : We denote by $\mathbf{T}_{a, b} \in \mathbb{C}^{N_{c} \times N_{d}}\left(\mathbf{t}_{a, b, c} \in \mathbb{C}^{N_{d}}\right.$, $t_{a, b, c, d} \in \mathbb{C}$ ) the matrix (vector, scalar) formed by slicing the tensor $\mathbf{T} \in \mathbb{C}^{N_{a} \times N_{b} \times N_{c} \times N_{d}}$ along the first two (three, four) dimensions. The notation $\mathbf{T}^{(k)}$ indicates that the quantity at hand is only considered for the $k^{\text {th }}$ user, and $\mathbf{v}_{-a}$ is the vector v without its $a^{\text {th }}$ element. $\mathbf{I}_{N}$ is the $N \times N$ identity matrix and $\mathbb{1}_{N \times M}$ is the $N \times M$ matrix with all elements set to 1 .

\section{Channel Model}

We consider a MU-MIMO system where $N_{k}$ single-antenna users transmit OFDM signals to a base station (BS) comprising $N_{m}$ antennas. The signals are transmitted over $N_{t}$ OFDM symbols and $N_{f}$ subcarriers, and the overall time-frequency grid is called resource grid (RG) and is illustrated in Fig. 1a. A resource element (RE) refers to one cell of the RG, and a group of 12 adjacent subcarriers is called a resource block. The channel corresponding to one $\mathrm{RE}(f, t)$ is denoted by $\mathbf{H}_{f, t} \in \mathbb{C}^{N_{m} \times N_{k}}$, and is a slice of the 4-dimensional tensor $\mathbf{H} \in \mathbb{C}^{N_{f} \times N_{t} \times N_{m} \times N_{k}}$ containing the channel coefficients of 


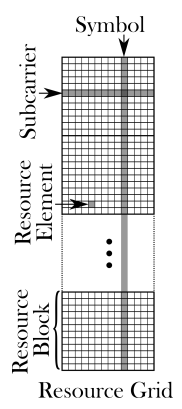

(a) Channel nomenclature.

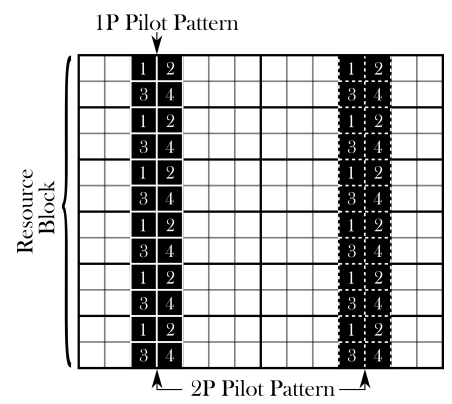

(b) $1 \mathrm{P}$ and $2 \mathrm{P}$ pilot patterns.
Fig. 1: Pilots are arranged on the RG following two distinct patterns, where the numbers represent different transmitters.

the entire RG. Following similar notations, the signal vectors sent by the users and received by the BS are respectively denoted by $\mathbf{x}_{f, t} \in \mathbb{C}^{N_{k}}$ and by $\mathbf{y}_{f, t} \in \mathbb{C}^{N_{m}} . M$ denotes the modulation order of the transmission. The channel transfer function on the $\operatorname{RE}(f, t)$ is expressed as

$$
\mathbf{y}_{f, t}=\mathbf{H}_{f, t} \mathbf{x}_{f, t}+\mathbf{n}_{f, t}
$$

where $\mathbf{n}_{f, t} \sim \mathcal{C N}\left(\mathbf{0}, \sigma^{2} \mathbf{I}_{N_{m}}\right)$ is the noise vector with a power $\sigma^{2}$ assumed equal for all users and all REs. It is assumed that all users have perfect power control such that the mean energy corresponding to a single user and receiving antenna is equal to one, i.e., $\mathbb{E}\left[\left|h_{f, t, k, m}\right|^{2}\right]=1$. The signal-to-noise ratio (SNR) of the transmission is defined as SNR $=10 \log \left(\frac{1}{\sigma^{2}}\right)[\mathrm{dB}]$.

Users both transmit data signals and pilot signals, the latter being assumed equal to one and are used by the BS to estimate the channel. Two pilot patterns are considered in this work, referred to as $1 \mathrm{P}$ and $2 \mathrm{P}$, and depicted in Fig. 1b. Each user $k$ sends pilots on a set of REs denoted by $\mathcal{P}^{(k)}$, and the number of OFDM symbols and subcarriers used to carry such pilots are respectively denoted by $N_{P_{t}}$ and $N_{P_{f}}$. For example, if only one resource block is considered and the $1 \mathrm{P}$ pilot pattern is used, then $\mathcal{P}^{(1)}=\{(1,3),(3,3),(5,3),(7,3),(9,3),(11,3)\}$, $N_{P_{t}}=1$, and $N_{P_{f}}=6$ (see Fig. 1b). The REs used by one user to transmit pilots are not used by the other users such that the pilots do not suffer from interferences.

\section{Conventional Receiver Architecture}

\section{A. Channel estimation}

The first step performed by a conventional receiver is to estimate the channel. As the pilots are orthogonal, channel estimation can be carried out separately for each user. The channel covariance matrix providing the spatial, temporal, and spectral correlation between all REs carrying pilots is denoted by $\boldsymbol{\Sigma} \in \mathbb{C}^{N_{P_{f}} N_{P_{t}} N_{m} \times N_{P_{f}} N_{P_{t}} N_{m}}$. This covariance matrix can be estimated by gathering a large dataset of received pilot signals, in contrast to the covariance between all REs that is usually not available in practice. To obtain the channel estimate $\widehat{\mathbf{H}}_{\mathcal{P}(k)}^{(k)}$ of user $k$ at REs carrying pilots, a well known estimator is the linear minimum mean squared error (LMMSE) filter:

$$
\operatorname{vec}\left(\widehat{\mathbf{H}}_{\mathcal{P}^{(k)}}^{(k)}\right)=\boldsymbol{\Sigma}\left(\boldsymbol{\Sigma}+\sigma^{2} \mathbf{I}\right)^{-1} \operatorname{vec}\left(\mathbf{Y}_{\mathcal{P}^{(k)}}^{(k)}\right)
$$

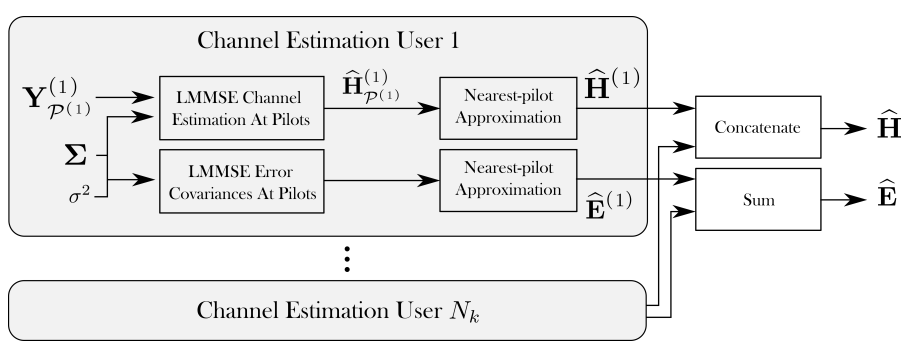

Fig. 2: Conventional channel estimation.

where $\mathbf{Y}_{\mathcal{P}(k)}^{(k)} \in \mathbb{C}^{N_{P_{f}} \times N_{P_{t}} \times N_{m}}$ is the tensor of received pilots for user $k$. The channel estimates for all REs are computed by first linearly interpolating the estimates from REs carrying pilots in the frequency dimension, and then using for each other REs the estimates computed at their nearest interpolated resource element (NIRE). It is also possible to leverage temporal linear interpolation when the $2 \mathrm{P}$ pilot pattern is used. The tensor of channel estimates for user $k$ is denoted by $\widehat{\mathbf{H}}^{(k)} \in \mathbb{C}^{N_{f} \times N_{t} \times N_{m}}$, and the tensor of channel estimates for all users $\widehat{\mathbf{H}} \in \mathbb{C}^{N_{f} \times N_{t} \times N_{k} \times N_{m}}$ is obtained by stacking all $\widehat{\mathbf{H}}^{(k)}$. The tensor of corresponding channel estimation errors is denoted by $\widetilde{\mathbf{H}} \in \mathbb{C}^{N_{f} \times N_{t} \times N_{k} \times N_{m}}$.

The spatial channel estimation error covariance is

$$
\mathbf{E}_{f, t}:=\sum_{k=1}^{N_{k}} \mathbf{E}_{f, t}^{(k)}=\sum_{k=1}^{N_{k}} \mathbb{E}\left[\tilde{\mathbf{h}}_{f, t}^{(k)} \tilde{\mathbf{h}}_{f, t}^{(k)} \mathrm{H}\right] \in \mathbb{C}^{N_{m} \times N_{m}}
$$

for the RE $(f, t)$, which is the sum of the spatial covariances $\mathbf{E}_{f, t}^{(k)}$ computed for each user. These covariance matrices only reflect the correlations between antennas, and not between different OFDM symbols or subcarriers. They are estimated on REs carrying pilots for each user independently, and shared by the groups of REs delimited by thick lines in Fig. 1b following a nearest-pilot approximation. The estimated error covariance for a $\operatorname{RE}(f, t)$ is denoted by $\widehat{\mathbf{E}}_{f, t} \in \mathbb{C}^{N_{m} \times N_{m}}$. The estimation procedure is shown in Fig. 2 and is further detailed in [11].

\section{B. Equalization and demapping}

Once the channel has been estimated, the equalization step aims to convert the MIMO channel on each RE into $N_{k}$ independent additive white Gaussian noise (AWGN) channels. Linear equalizers such as LMMSE are widely used because of their reasonable complexity. However, the standard LMMSE operator requires the computation of a matrix inversion per $\mathrm{RE}$, which can be too computationally expensive in large systems. Therefore, we leverage a grouped-LMMSE equalizer, where a single operator $\mathbf{W}_{f, t} \in \mathbb{C}^{N_{k} \times N_{m}}$ is computed and applied to a group of REs spanning $\left\{F_{b}, \ldots, F_{e}\right\} \times\left\{T_{b}, \ldots, T_{e}\right\}$, as delimited in Fig. 1b. Assuming perfect knowledge of $\mathbf{E}$, such operator is computed as (see [11] for a derivation)

$$
\begin{aligned}
\mathbf{W}_{f, t}= & \left(\sum_{f^{\prime}=F_{b}}^{F_{e}} \sum_{t^{\prime}=T_{b}}^{T_{e}} \widehat{\mathbf{H}}_{f^{\prime}, t^{\prime}}^{\mathrm{H}}\right) \\
& \left(\sum_{f^{\prime}=F_{b}}^{F_{e}} \sum_{t^{\prime}=T_{b}}^{T_{e}} \widehat{\mathbf{H}}_{f^{\prime}, t^{\prime}} \widehat{\mathbf{H}}_{f^{\prime}, t^{\prime}}^{\mathrm{H}}+\mathbf{E}_{f^{\prime}, t^{\prime}}+\sigma^{2} \mathbf{I}_{N_{m}}\right)^{-1} .
\end{aligned}
$$




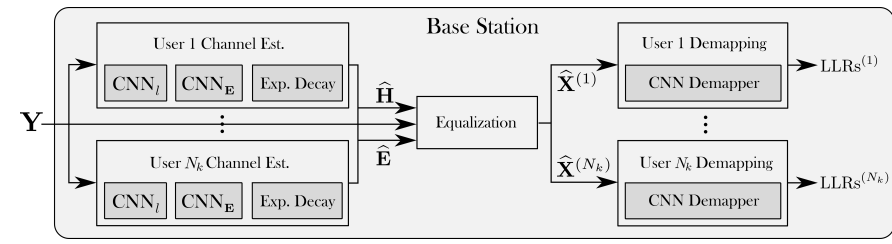

Fig. 3: ML-enhanced receiver architecture.

In order to obtain $N_{k}$ AWGN channels, the equalized symbols $\hat{\mathbf{x}}_{f, t}$ needs to be scaled such that $\hat{x}_{f, t, k}=x_{f, t, k}+z_{f, t, k}$, where $z_{f, t, k} \sim \mathcal{C N}\left(0, \rho_{f, t, k}^{2}\right)$ includes the interference and noise of user $k .{ }^{1}$ The corresponding scaling matrix

$$
\mathbf{D}_{f, t}=\left(\left(\mathbf{W}_{f, t} \hat{\mathbf{H}}_{f, t}\right) \odot \mathbf{I}_{N_{k}}\right)^{-1}
$$

is thus applied to the equalized signals, i.e.,

$$
\hat{\mathbf{x}}_{f, t}=\mathbf{D}_{f, t} \mathbf{W}_{f, t} \mathbf{y}_{f, t}
$$

and the post-equalization noise variance $\rho_{f, t, k}^{2}$ is given by

$$
\rho_{f, t, k}^{2}=\frac{\mathbf{w}_{f, t, k}^{\mathrm{H}}\left(\widehat{\mathbf{H}}_{f, t,-k} \widehat{\mathbf{H}}_{f, t,-k}^{\mathrm{H}}+\mathbf{E}_{f, t}+\sigma^{2} \mathbf{I}_{N_{m}}\right) \mathbf{w}_{f, t, k}}{\mathbf{w}_{f, t, k}^{\mathrm{H}} \widehat{\mathbf{h}}_{f, t, k} \widehat{\mathbf{h}}_{f, t, k}^{\mathrm{H}} \mathbf{w}_{f, t, k}} .
$$

Log-likelihood ratios (LLRs) are obtained using a standard AWGN demapper on each user $k$ and $\operatorname{RE}(f, t)$ independently, assuming a post-equalization noise variance of $\rho_{f, t, k}^{2}$. Finally, $\mathbf{E}_{f, t}$ is often not available and is replaced by its estimate $\hat{\mathbf{E}}_{f, t}$.

\section{ML-ENHANCED RECEIVER ARCHITECTURE}

\section{A. Receiver training}

The proposed ML-enhanced receiver is depicted in Fig. 3, where the grayed elements represent trainable components. In contrast to many related papers that optimize each receive processing block separately, we chose to train all ML components together to optimize the estimated LLRs. This approach is more practical since it does not assume that ground-truth channel measurements are available. The set of all trainable parameters $\boldsymbol{\theta}$ is optimized with stochastic gradient descent on the binary cross-entropy loss and using batches of $B_{s}$ samples:

$$
\mathcal{L}=-\frac{1}{B_{s}} \sum_{s=1}^{B_{s}} \sum_{k=1}^{N_{k}} \sum_{(f, t) \in \mathcal{D}} \sum_{m=1}^{M} \log _{2}\left(\widetilde{P}_{\boldsymbol{\theta}}\left(b_{f, t, k, m}^{[s]} \mid \mathbf{Y}^{[s]}\right)\right)
$$

where $\mathcal{D}$ denotes the set of REs carrying data, $b_{f, t, k, m}$ the $m^{\text {th }}$ bit of user $k$ on the RE $(f, t)$, and the superscript $[s]$ refers to the $s^{\text {th }}$ sample in the batch. $\widetilde{P}_{\boldsymbol{\theta}}\left(b_{f, t, k, m}^{[s]} \mid \mathbf{Y}^{[s]}\right)$ is the estimated posterior probabilities on the bit $b_{f, t, k, m}^{[s]}$ and is obtained by applying the sigmoid function to the corresponding LLRs. As detailed in [10], minimizing this loss is equivalent to maximizing the sum of the achievable rates for all users.

\section{B. ML-enhanced channel estimator}

The NIRE approximation used by the conventional channel estimator of Section III implies that the estimation of the

\footnotetext{
${ }^{1}$ This is not true in general as the interference and channel estimation errors are not Gaussian distributed.
}

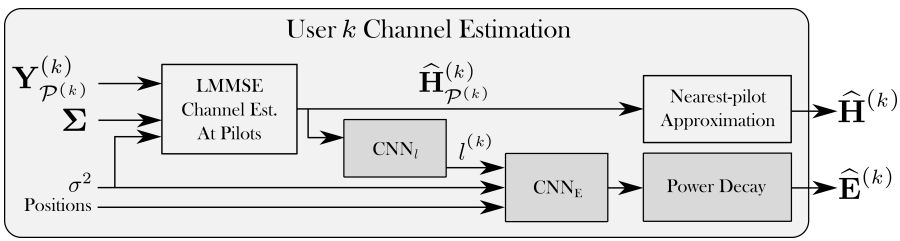

Fig. 4: ML-enhanced channel estimation of user $k$.

covariances $\mathbf{E}_{f, t}$ might be inaccurate at REs that are far from pilots. This in turn causes the computation of $\mathbf{W}_{f, t}$ in (4) and of $\rho_{f, t, k}^{2}$ in (7) to be erroneous. In the following, we present a suite of ML components, represented in grey in Fig. 4, that aim to better predict these channel estimation error covariances.

Fig. 5 shows an example of the amplitudes and phases of an error covariance matrix $\mathbf{E}_{f, t}^{(k)}$. Predicting the complex coefficient of $\mathbf{E}_{f, t}$ for all REs would be of prohibitive complexity for a naive NN. To tackle this problem, we approximate every element $(x, y)$ of $\mathbf{E}_{f, t}$ with a complex power decay model:

$$
\hat{e}_{f, t, x, y}^{(k)}=\alpha_{f, t} \beta_{f, t}^{|y-x|} \exp (j \gamma(y-x))
$$

where $\alpha_{f, t}$ and $\beta_{f, t}$ are parameters that control the scale and the decay of the model, and are different for every RE $(f, t)$. We observed that the phase offset $\gamma$ was close to $\pi$ and constant over the RG, and is therefore implemented as a single trainable parameter that will be optimized according to (8).

To estimate $\alpha_{f, t}$ and $\beta_{f, t}$ for all REs, we propose to use a CNN, denoted by $\mathrm{CNN}_{\mathbf{E}}$. This $\mathrm{CNN}$ has an output of dimension of $N_{f} \times N_{t} \times 2$, corresponding to the two parameters being predicted on the entire $\mathrm{RG}$, and has four inputs of dimension $N_{f} \times N_{f}$, for a total input dimension of $N_{f} \times N_{f} \times 4$. CNNs are known to be translation invariant, however the predictions $\alpha_{f, t}$ and $\beta_{f, t}$ depend on the position $(f, t)$ in the RG. Therefore, vertical and horizontal positional information are given to $\mathrm{CNN}_{\mathbf{E}}$ by the first two input matrices, that respectively have all columns equal to $\left[-\frac{N_{f}}{2}, \cdots,-1,1, \cdots, \frac{N_{f}}{2}\right]^{\top}$ and all rows equal to $\left[\frac{N_{t}}{2}, \cdots,-1,1, \cdots, \frac{N_{t}}{2}\right]$. The third input is the SNR of the transmission, given as SNR $\mathbb{1}_{N_{f} \times N_{t}}$. The fourth input is designed to provide information about the time and frequency selectivity of the channel, since the NIRE approximation of $\widehat{\mathbf{H}}^{(k)}$ is likely to be less precise on fast-varying channels.

This time-variability information is in turn estimated by a second CNN, denoted by $\mathrm{CNN}_{l}$. The goal of this $\mathrm{CNN}$ is to extract a feature related to the Doppler and delay spread of the transmission from the estimated channel at REs carrying pilots. This feature is a single scalar denoted by $l^{(k)} \in \mathbb{R}$ for

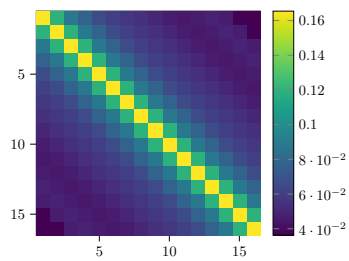

(a) Amplitude

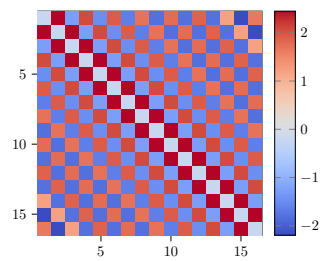

(b) Phase
Fig. 5: Example of amplitude and phase for $\mathbf{E}_{f, t}^{(k)}$. 


\begin{tabular}{|c|c|c|c|c|c|c|}
\hline & \multicolumn{3}{|c|}{$\mathrm{CNN}_{l}$} & \multicolumn{3}{|c|}{$\mathrm{CNN}_{\mathrm{Dmp}}$} \\
\hline Parameters & filters & kernel & dilat. & filters & kernel & dilat. \\
\hline Conv2D & 32 & $(1,1)$ & $(1,1)$ & 128 & $(1,1)$ & $(1,1)$ \\
\hline ResNet Layer & 32 & $(3,2)$ & $(1,1)$ & 128 & $(3,3)$ & $(1,1)$ \\
\hline ResNet Layer & 32 & $(5,2)$ & $(2,1)$ & 128 & $(5,3)$ & $(2,1)$ \\
\hline ResNet Layer & 32 & $(7,2)$ & $(3,1)$ & 128 & $(7,3)$ & $(3,2)$ \\
\hline ResNet Layer & 32 & $(5,2)$ & $(2,1)$ & 128 & $(9,3)$ & $(4,3)$ \\
\hline ResNet Layer & 32 & $(3,2)$ & $(1,1)$ & 128 & $(7,3)$ & $(3,2)$ \\
\hline ResNet Layer & \multicolumn{3}{|c|}{ - } & 128 & $(5,3)$ & $(2,1)$ \\
\hline ResNet Layer & \multicolumn{3}{|c|}{ - } & 128 & $(3,3)$ & $(1,1)$ \\
\hline Conv2D & 1 & $(3,2)$ & $(1,1)$ & $M$ & $(1,1)$ & $(1,1)$ \\
\hline Output Layer & \multicolumn{3}{|c|}{ Dense, units $=1$} & \multicolumn{3}{|c|}{ - } \\
\hline
\end{tabular}

TABLE I: Architectures of the different CNNs.

user $k$, as shown in Fig. 4. $\mathrm{CNN}_{l}$ has inputs of dimension $N_{P_{f}} \times N_{P_{t}} \times 2 N_{m}$, corresponding to the stacking of the real and imaginary part of $\widehat{\mathbf{H}}_{\mathcal{P}^{(k)}}^{(k)}$, and therefore outputs a single scalar. Finally, $l^{(k)}$ is fed to $\mathrm{CNN}_{\mathbf{E}}$ as the matrix $l^{(k)} \cdot \mathbb{1}_{N_{f} \times N_{t}}$. The architecture of all CNNs are detailed in Section IV-D.

\section{ML-enhanced demapper}

The sub-optimal channel estimation and equalization create distortions in the equalized signals. As seen in Section III, a conventional demapper processes each user and RE independently. In this work, we take a different approach and propose a new CNN-based demapper, denoted by $\mathrm{CNN}_{\mathrm{Dmp}}$, that operates directly on the two-dimensional RG. The demapping is still applied to each user independently (see Fig.3), preserving the scalability of the conventional architecture, but the joint processing of all REs allows $\mathrm{CNN}_{\text {Dmp }}$ to estimate and correct the distortions present in the equalized signals. $\mathrm{CNN}_{\text {Dmp }}$ takes six inputs, each of size $N_{f} \times N_{t}$. Similarly to $\mathrm{CNN}_{\mathbf{E}}$, the first two inputs are the positioning matrices, and the third input is the SNR. The forth input contains the post-equalization noise variances $\rho_{f, t, k}^{2}$ for all REs $(f, t)$. The fifth and sixth inputs are the real and imaginary parts of the equalized symbols $\widehat{\mathbf{X}}^{(k)}$. The output of $\mathrm{CNN}_{\mathrm{Dmp}}$ is of dimension $N_{f} \times N_{t} \times M$, corresponding to the LLRs of user $k$.

\section{CNN architectures}

$\mathrm{CNN}_{\mathrm{E}}, \mathrm{CNN}_{l}$, and $\mathrm{CNN}_{\text {Dmp }}$ share similar architectures. $\mathrm{CNN}_{\mathbf{E}}$ uses two convolutional 2D layers with 32 filters, kernel sizes of $(5,3)$, dilation rates of $(1,1)$, zero-padding, and rectified linear unit (ReLU) activation functions. Its output layer is a convolutional 2D layer but with only two filters, a kernel size and dilation rate of $(1,1)$, zero-padding, and sigmoid activation function. Both $\mathrm{CNN}_{l}$ and $\mathrm{CNN}_{\mathrm{Dmp}}$ use custom ResNet layers, inspired by [12], and composed of a batch normalization layer, a ReLU, a separable convolutional 2D layer, and followed by the addition of the input, as shown in Fig. 6. Separable convolutional 2D layers are known to be more efficient than traditional convolutional layers [13]. The architecture of $\mathrm{CNN}_{l}$ and $\mathrm{CNN}_{\text {Dmp }}$ are detailed in Table I, where all convolutional layers use zero-padding to keep consistent dimensions. Varying kernel sizes and dilation rates were used to increase the receptive field of the CNNs [9].

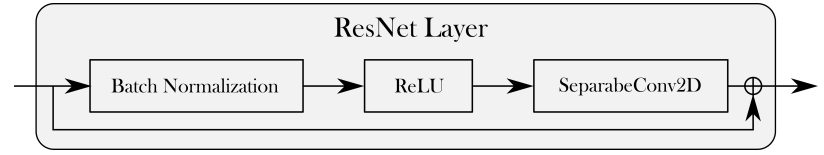

Fig. 6: Custom ResNet layer.

\section{Evaluations}

\section{A. Training and evaluation setup}

The number of users and receive antennas were set to $N_{k}=4$ and $N_{m}=16$, respectively. Each user was randomly positioned between 15 and $150 \mathrm{~m}$ of the BS in a $120^{\circ}$ cell sector. The users and BS heights were respectively set to $1.5 \mathrm{~m}$ and $10 \mathrm{~m}$. Realistic channel realizations were generated with QuaDRiGa version 2.0.0 using the 3GPP non-line of sight (NLOS) urban microcell (UMi) channel model. The RGs were composed of $N_{t}=14$ OFDM symbols and $N_{f}=72$ subcarriers (six resource blocks). The center frequency was set to $3.5 \mathrm{GHz}$ and the subcarrier spacing to $15 \mathrm{kHz}$. A Graylabeled 16-QAM was used to transmit $M=4$ bits per symbol. Six ranges of user speed were considered: 0 to $15 \mathrm{~km} \mathrm{~h}^{-1}, 15$ to $30 \mathrm{~km} \mathrm{~h}^{-1}$, and 30 to $45 \mathrm{~km} \mathrm{~h}^{-1}$ for the $1 \mathrm{P}$ pilot pattern, and 50 to $70 \mathrm{~km} \mathrm{~h}^{-1}, 80$ to $100 \mathrm{~km} \mathrm{~h}^{-1}$, and 110 to $130 \mathrm{~km} \mathrm{~h}^{-1}$ for the $2 \mathrm{P}$ pilot pattern. We noticed that $\mathrm{CNN}_{l}$ was not able to extract useful information from the $1 \mathrm{P}$ pattern, and therefore was not used in the corresponding training and evaluations.

Two separate sets were considered to train the ML-enhanced receiver on the $1 \mathrm{P}$ and $2 \mathrm{P}$ pilot patterns. Both training sets were made of $3000 \mathrm{RGs}$ constructed from $1000 \mathrm{RGs}$ of the three users speed ranges for each pilot pattern. The receiver parameters were randomly initialized, except for $\gamma$ that was initialized at $\pi$. The Adam optimizer was used for training, with a batch size of $B_{s}=27$ and a learning rate of $10^{-3}$. During evaluations, a standard IEEE 802.11n low-density parity-check (LDPC) code of length 1296 bit was used in conjunction with 40 iterations of a conventional belief-propagation decoder. To satisfy the perfect power allocation assumption, the average energy per user and antenna on the RGs were normalized such that $\sum_{f=1}^{N_{f}} \sum_{t=1}^{N_{t}}\left\|\mathbf{h}_{f, t}^{(k)}\right\|_{2}^{2}=N_{f} N_{t} N_{m}$.

\section{B. Simulation results}

The proposed ML-enhanced receiver was compared against two systems. The first one is the conventional receiver baseline detailed in Section III. The second one, referred to as 'Perfect CSI receiver', has the same architecture but has access to perfect knowledge of the channel at REs carrying pilots and to $\mathbf{E}_{f, t}$ at every RE, obtained from Monte-Carlo simulations. Additional simulations were conducted for the $2 \mathrm{P}$ pilot pattern using spectral and temporal interpolation for both the baseline and the ML receiver. Finally, an ML receiver trained with only $N_{k}=2$ users was also evaluated with $N_{k}=4$ users. The first row of Fig. 7 presents evaluation results corresponding to the 1P pattern. It can be seen that at low speed ( 0 to $\left.15 \mathrm{~km} \mathrm{~h}^{-1}\right)$, the ML-enhanced receiver achieves a $0.8 \mathrm{~dB}$ gain over the conventional receiver at a coded bit error rate (BER) of $10^{-3}$, but is still $2.3 \mathrm{~dB}$ behind the perfect CSI receiver. Between 35 and $45 \mathrm{~km} \mathrm{~h}^{-1}$, the conventional receiver saturates at high 


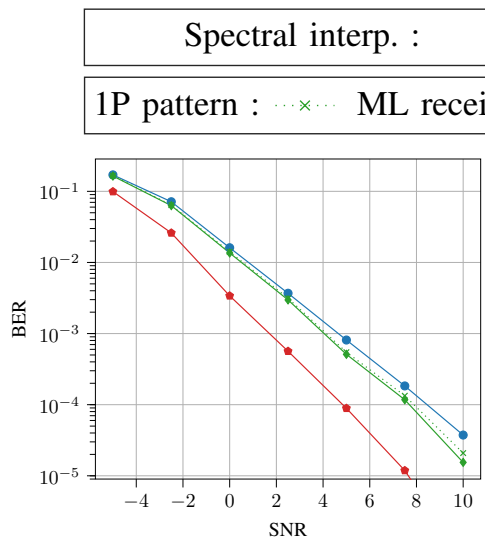

(a) $1 \mathrm{P}$ pilot pattern at 0 to $15 \mathrm{~km} \mathrm{~h}^{-1}$.

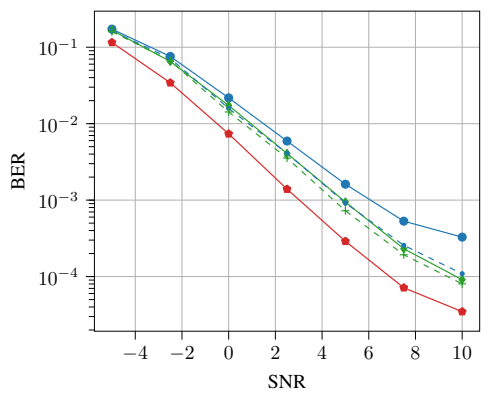

(d) $2 \mathrm{P}$ pilot pattern at 50 to $70 \mathrm{~km} \mathrm{~h}^{-1}$.

Baseline

ML receiver

$\rightarrow$

Perfect CSI

2P pattern, dual interp. : - - Baseline - +- ML rec.

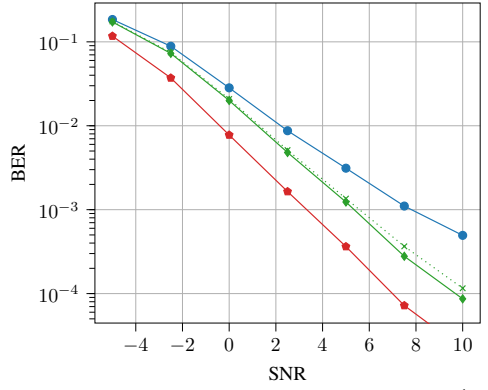

(b) $1 \mathrm{P}$ pilot pattern at 15 to $30 \mathrm{~km} \mathrm{~h}^{-1}$.

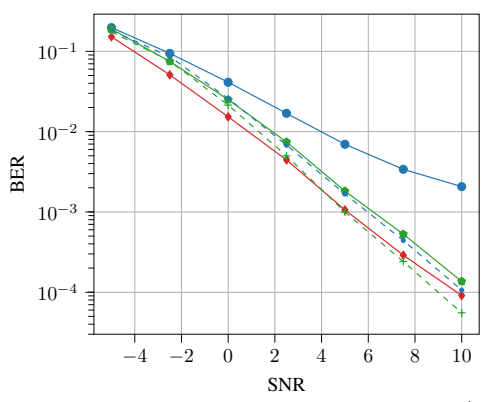

(e) $2 \mathrm{P}$ pilot pattern at 80 to $100 \mathrm{~km} \mathrm{~h}^{-1}$.

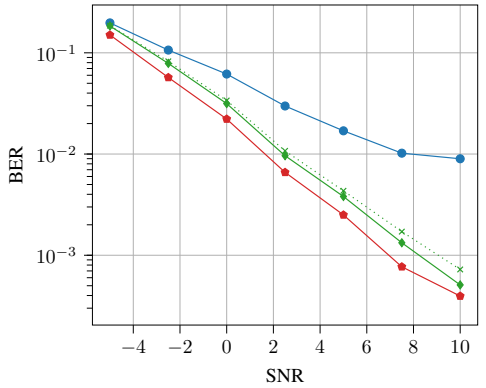

(c) $1 \mathrm{P}$ pilot pattern at 30 to $45 \mathrm{~km} \mathrm{~h}^{-1}$.

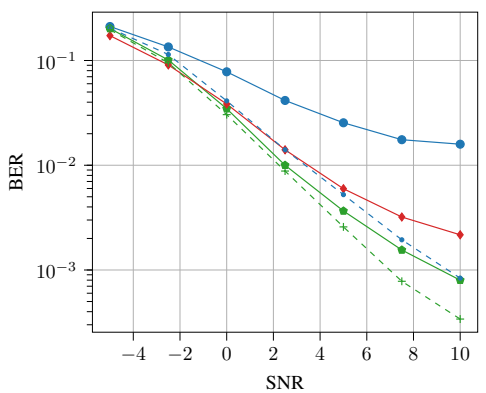

(f) $2 \mathrm{P}$ pilot pattern at 110 to $130 \mathrm{~km} \mathrm{~h}^{-1}$.

Fig. 7: Uplink BER achieved by the different receivers with the 1P and 2P pilot patterns.

SNR while the gap between the ML-enhanced and perfect CSI receivers is narrowing, leading to a gain of $2.5 \mathrm{~dB}$ at a BER of $10^{-2}$. The ML receiver trained with a mismatched number of users only suffers a small performance drop, demonstrating the scalability of the ML scheme. The $2 \mathrm{P}$ pattern results shown in the second row of Fig. 7 follow the same trend, with increasing gains at higher speeds. In the 110 to $130 \mathrm{~km} \mathrm{~h}^{-1}$ range, the ML-enhanced receiver even outperforms the perfect CSI baseline, which suffers from strong channel aging that the CNN demapper is able to mitigate. Using both spectral and temporal interpolations reduces the gains provided by the ML receiver, which can be explained by the better channel estimates leading to less channel aging, but they still amount to a $2.2 \mathrm{~dB}$ gap at a BER of $10^{-3}$ for the highest speeds. More detailed uplink and downlink evaluation results can be found in [11], including a more detailed analysis on the interpretability and usefulness of each ML component.

\section{CONCLUSION}

In this paper, we have enhanced a conventional MU-MIMO receiver architecture using multiple CNNs to improve both the computation of its channel estimation error statistics and of the estimated bit probabilities. Compared to other approaches, all ML components of the receiver are jointly optimized to maximize the information rate of the transmission, which does not require any ground truth measurements. Evaluation results indicate that the ML receiver achieves gains across all scenarios, and especially at high speeds, while preserving the scalability and interpretability of conventional architectures.

\section{REFERENCES}

[1] K. B. Letaief, W. Chen, Y. Shi, J. Zhang, and Y. A. Zhang, "The Roadmap to 6G: AI Empowered Wireless Networks," IEEE Commun. Mag., vol. 57, no. 8, pp. 84-90, Aug 2019.

[2] J. Downey, B. Hilburn, T. O'Shea, and N. West, "Machine learning remakes radio," IEEE Spectrum, vol. 57, no. 5, pp. 35-39, 2020.

[3] P. Dong, H. Zhang, G. Y. Li, I. S. Gaspar, and N. NaderiAlizadeh, "Deep CNN-Based Channel Estimation for mmWave Massive MIMO Systems," IEEE J. Sel. Topics Signal Process., vol. 13, no. 5, pp. 989-1000, 2019.

[4] M. B. Mashhadi and D. Gunduz, "Pruning the Pilots: Deep LearningBased Pilot Design and Channel Estimation for MIMO-OFDM Systems," preprint arXiv:2006.11796, 2020.

[5] K. Pratik, B. D. Rao, and M. Welling, "RE-MIMO: Recurrent and Permutation Equivariant Neural MIMO Detection," preprint arXiv:2007.00140, 2020.

[6] H. He, C. Wen, S. Jin, and G. Y. Li, “A Model-Driven Deep Learning Network for MIMO Detection," in Proc. IEEE Global Conf. Signal Inf. Process. (GlobalSIP), 2018, pp. 584-588.

[7] M. Khani, M. Alizadeh, J. Hoydis, and P. Fleming, "Adaptive Neural Signal Detection for Massive MIMO," arXiv:1906.04610, Jun 2019.

[8] O. Shental and J. Hoydis, ""Machine LLRning": Learning to Softly Demodulate," preprint arXiv:1907.01512, 2020.

[9] D. Korpi, M. Honkala, J. M. J. Huttunen, and V. Starck, "DeepRx MIMO: Convolutional MIMO Detection with Learned Multiplicative Transformations," preprint arXiv:2010.16283, 2020.

[10] S. Cammerer, F. A. Aoudia, S. Dörner, M. Stark, J. Hoydis, and S. ten Brink, "Trainable Communication Systems: Concepts and Prototype," IEEE Trans. Commun., vol. 68, no. 9, pp. 5489-5503, 2020.

[11] M. Goutay, F. Ait Aoudia, J. Hoydis, and J.-M. Gorce, "Machine Learning for MU-MIMO Receive Processing in OFDM Systems," preprint arXiv:2012.08177, 2020.

[12] K. He, X. Zhang, S. Ren, and J. Sun, "Identity mappings in deep residual networks," in Eur. Conf. Comput. vision. Springer, 2016, pp. 630-645.

[13] A. G. Howard, M. Zhu, B. Chen, D. Kalenichenko, W. Wang, T. Weyand, M. Andreetto, and H. Adam, "MobileNets: Efficient Convolutional Neural Networks for Mobile Vision Applications," preprint arXiv:1704.04861, 2017. 